\title{
Routine parallel diagnosis of malaria using microscopy and the malaria rapid diagnostic test SD 05FK60: the experience of Médecins Sans Frontières in Myanmar
}

\author{
Cara S Kosack ${ }^{1 *}$, Wint Thu Naing ${ }^{2}$, Erwan Piriou ${ }^{3}$ and Leslie Shanks ${ }^{3}$
}

\begin{abstract}
Background: Malaria rapid diagnostic tests (RDTs) are commonly used in Médecins Sans Frontières (MSF) programmes to detect acute malaria infection. Programmes in regions with both Plasmodium falciparum and non-falciparum malaria (i.e. Plasmodium ovale, Plasmodium malariae and Plasmodium vivax) use a three-band P. falciparum/Pan test such as the SD Bioline Malaria Ag P.f/Pan 05FK60 (Standard Diagnostics, Kyonggi, Republic of Korea), hereafter referred to as SD 05FK60, as used by the MSF-Holland clinics in Rakhine state, Myanmar. In spite of published reports of generally good test performance, medical and paramedical staff on the ground often doubt the diagnostic accuracy of these RDTs.
\end{abstract}

Methods: Parallel testing with malaria microscopy and RDT was conducted at two clinics in Rakhine state, Myanmar, for a period of 14 months as a programmatic response due to doubts and concerns of medical and paramedical staff into malaria RDTs.

Results: A total of 2,585 blood samples from non-pregnant suspected malaria patients were examined by the SD 05FK60 RDT and microscopy at two clinics in Myanmar from October 2010 to December 2011. The reference standard microscopy diagnosed 531 P. falciparum and 587 P. vivax or P. malariae mono-infections. The overall sensitivity for $P$. falciparum detection by the SD 05FK60 was $90.2 \%$ (95\% Cl: 87.4-92.6) and for $P$. vivax/P. malariae 79.4\% (95\% Cl: 75.9-82.6). The overall specificity for P. falciparum detection by the SD 05FK60 was 98.5\% (95\% Cl: $97.7-99.1$ ) and for P. vivax/P. malariae $98.7 \%$ (95\% Cl: 97.9-99.2). The sensitivity for $P$. falciparum was $>91 \%$ for parasitaemia levels of $>100-1,000$ parasites/ $\mu$ and increased for $P$. vivax/P. malariae with the parasitaemia level but was overall lower than for $P$. falciparum 25/408 and 13/420 cases, respectively, of $P$. falciparum and non-falciparum malaria were missed by the RDT.

Conclusion: In field conditions in Myanmar, the SD 05FK60 malaria RDT performed consistent with other reports. The test detected malaria caused by $P$. vivax/P. malariae to a lesser extent than $P$. falciparum infection. Sensitivity improved with increasing parasitaemia level, however even at higher levels some infections were missed. The SD 05FK60 is adequate for use in settings where high quality microscopy is not available.

Keywords: Malaria, RDT, Rapid diagnostic test, Sensitivity, Specificity, Diagnostic test accuracy, Parasite lactate dehydrogenase tests, Plasmodium vivax, Plasmodium falciparum, SD 05FK60

\footnotetext{
* Correspondence: cara.kosack@amsterdam.msf.org

'Médecins Sans Frontières International, Plantage Middenlaan 14, 1018 DD Amsterdam, The Netherlands

Full list of author information is available at the end of the article
} 


\section{Background}

Microscopy is the reference standard for diagnosing malaria but is time consuming and requires considerable training and experience. It is ill suited as a diagnostic tool in settings where the case load is high and resources limited [1,2]. Lateral-flow immunoassays (LFIs), often called rapid diagnostic tests (RDTs), are an attractive alternative as they are quick and easy to carry out [1-7]. In practice however, malaria is often diagnosed solely on the basis of clinical symptoms, without using either method; this results in over-treatment of malaria and the risk of missing true underlying pathologies. To improve the accuracy of malaria diagnosis and avoid unnecessary treatment, the World Health Organization (WHO) recommends parasitological confirmation of malaria whenever possible, by RDT or by microscopy $[1,8-11]$.

Among the newer generation of malaria RDTs are three-band tests that detect both the Plasmodium falciparum-specific antigen histidine-rich protein 2 (HRP2) and the pan-Plasmodium-specific lactate dehydrogenase (pLDH), expressed in the four most common malaria parasite species [P. falciparum, Plasmodium ovale, Plasmodium malariae and Plasmodium vivax [6,12-16].

In 2009, Médecins Sans Frontières (MSF) introduced routine use of HRP2/pan-pLDH RDTs into its programmes at sites where both $P$. falciparum and nonfalciparum malaria are present (i e, Southeast Asia, the Americas and the Ethiopian highlands). In Myanmar, which has an enormous malaria burden, accounting for 7\% of all malaria cases in Southeast Asia and India [7], $P$. vivax is second only to $P$. falciparum as the cause of malaria, so the test chosen was the SD Bioline 05F K60 Malaria Ag P.f/Pan (Standard Diagnostics, Kyonggi, Republic of Korea), hereafter referred to as SD 05FK60.

The choice was made for the SD 05FK60 because it was one of the best performing assays in the WHO/TDR (Special Programme for Research and Training in Tropical Diseases)/FIND (Foundation for Innovative New Diagnostics)/CDC (Centers for Disease Control) evaluation in Round 1 and because continuous supply and importation to Myanmar could be guaranteed. However, since 2007, evaluations of the SD 05FK60 were conducted in returned travellers or as mentioned before by the WHO/TDR/ FIND/CDC programme but not on a field site. In these evaluations, the SD 05FK60 showed good performance for detection of malaria caused by $P$. falciparum, with varying results for $P$. vivax detection [17-22].

However, in spite of the generally good performance of malaria RDTs, their use on the ground can be problematic: medical staff frequently mistrust the results and problems with the products (e g, poor migration of blood and buffer, faint lines, incorrect placement of the lateral-flow strip in the cassette) are encountered regularly [23-25]. This often results in low coverage of RDT implementation and poor adherence to RDT results (as the basis for treatment decisions) in clinical practice [26]. In order to monitor the performance of this newly introduced RDT in MSF's programmes in Myanmar and to respond to clinicians' concerns of poor RDT performance, it was decided to test patients in parallel with microscopy and RDT until the end of 2011 in our programmes in Rakhine State. In Myanmar, approximately $68 \%$ of the population of over 60 million total, is thought to be at risk for malaria with the high risk areas concentrated near international borders, including Rakhine state. The yearly burden in the entire country is to be over two million cases [16].

\section{Methods}

\section{Investigation sites and procedures}

MSF has been working in Myanmar's Rakhine State since 1993. Primary health care (PHC), including the diagnosis and treatment of malaria, is provided free of charge at two clinics, in Sittwe and Thetkalpyin, located in the eastern region of Rakhine. All patients presenting at the Sittwe and Thetkalpyin clinics with fever or a history of fever in the prior 24 hours were immediately tested for malaria on capillary blood with the SD 05FK60 RDT. In addition, a slide was prepared from the same capillary sample and examined separately in the laboratory regardless of the SD 05FK60 result. The laboratory technician was not aware of the SD 05FK60 result when examining the smear. Pregnant women screened for malaria as part of antenatal care received the same procedure but were not included in this analysis.

This routine parallel testing of the described population was carried out from 25 October, 2010 until 23 December, 2011 with one month of data lost (23 February to 28 March 2011) as a result of security incidents in eastern Rakhine State.

\section{Microscopy}

Thick and thin blood films were prepared and stained with $10 \%$ Giemsa solution ( $\mathrm{pH} 7.2)$ for $10 \mathrm{~min}$ and examined by light microscopy using x1,000 magnification. At least 200 fields had to be examined before designating a sample 'negative', or more accurately, 'no malaria parasites seen'. Positive findings were graded on the thick smear using the 'plus' system scale: + (1 to 9 trophozoites in 100 fields); ++ (1 to 10 trophozoites in 10 fields); +++ ( 1 to 10 trophozoites per field); $++++(>10$ trophozoites per field). These scores were used to estimate parasite densities: $+=10$ to 90 parasites $/ \mu \mathrm{l} ;++=100$ to 1,000 parasites $/ \mu \mathrm{l},+++=1,000$ to 10,000 parasites $/ \mu \mathrm{l} ;++++=>$ 10,000 parasites $/ \mu \mathrm{l}$, assuming a white blood cell count of $8,000 / \mu \mathrm{l}$. The species was identified using the thin smear. 


\section{Rapid diagnostic test}

The SD 05FK60 RDT is a three-band lateral-flow immunochromatographic antigen detection test in a cassette format and testing was carried out according to the manufacturer's instructions. Readings were taken in daylight, assisted by standard electric lighting. A negative result is indicated by the presence of a single line, the ' $C$ ' control line, in the result window. A P. falciparum-positive result was indicated by presence of two colour bands, the $P$. falciparum test line and the ' $C$ ' control line. The presence of the 'Pan' test line and the ' $C$ ' control line (again, two colour bands) indicated a $P$. vivax, $P$. ovale. or $P$. malariae-positive result or a mixed non-falciparum infection. The presence of three colour bands, the ' $P$. falciparum' and 'Pan' test lines and the ' $\mathrm{C}$ ' control line indicates a $P$. falciparum-positive result or a mixed infection. In cases where the control line did not appear, the results were interpreted as invalid and the test repeated with a new device.

\section{Statistical analysis}

Data were entered in an Excel file and analysed using Stata 11.0 statistical software (Stata Corporation, College Station, Texas, USA). Samples with pure gametocytaemia were considered negative. Microscopically identified mixed infections were considered separately (Table 1) and not included in the calculations of test accuracy (Table 2). Sensitivity, specificity and predictive values were calculated with 95\% confidence intervals (CI) for the detection of $P$. falciparum and non-falciparum species separately. When calculating the performance parameters for $P$. falciparum all negative samples plus all $P$. falciparum positive samples by microscopy contributed to the analysis. Similarly for the non-falciparum analysis all negative samples and all nonfalciparum positive samples by microscopy contributed to the analysis. Likelihood ratios were calculated using the following formulae: $\mathrm{LR}+=$ sensitivity/(1-specificity) and LR $-=(1-$ specificity)/sensitivity (Table 2$)$.

\section{Quality control}

All malaria RDTs purchased by MSF must pass the WHO lot-testing programme before being shipped to field sites
Table 2 Performance of SD Bioline Malaria Ag P.f/Pan (05FK60) rapid diagnostic test (mixed infections excluded)

\begin{tabular}{lll}
\hline $\begin{array}{l}\text { Microscopy } \\
\text { results }\end{array}$ & $\begin{array}{l}\text { P. falciparum } \\
\mathbf{N}=\mathbf{1 , 8 6 8} \\
\text { (531 positive) }\end{array}$ & $\begin{array}{l}\text { P. vivax/P. malariae } \\
\mathbf{N}=\mathbf{1 , 9 2 4} \\
\text { (587 positive) }\end{array}$ \\
\hline Sensitivity (95\% Cl) & $90.2(87.4-92.6)$ & $79.4(75.9-82.6)$ \\
Specificity (95\% Cl) & $98.5(97.7-99.1)$ & $98.7(97.9-99.2)$ \\
PPV (95\% Cl) & $96.0(93.9-97.5)$ & $96.3(94.2-97.8)$ \\
NPV (95\% Cl) & $96.2(95.0-97.2)$ & $91.6(90.0-93.0)$ \\
LR+ (95\% Cl) & $60.3(39-93.2)$ & $59.0(37.2-93.5)$ \\
LR- $\mathbf{( 9 5 \% ~ C l ) ~}$ & $0.10(0.08-0.13)$ & $0.21(0.18-0.25)$
\end{tabular}

A total of 2,585 blood samples was examined at the Sittwe and Thetkalpyin clinics, Rakhine State, Myanmar.

PPV: positive predicted value; NPV: negative predictive value; LR: likelihood ratio; LR+: sensitivity/(1-specificity); LR-: (1-specificity)/sensitivity.

[27]. In addition, the programme follows the WHO Malaria Microscopy Quality Assurance recommendation [28]. In accordance with this protocol, slides were sent to the Shoklo Malaria Research Unit (SMRU) in Mae Sot, Thailand, for external quality control.

\section{Ethical statement}

This manuscript reports a descriptive retrospective analysis of routinely collected data, and therefore no protocol was submitted for ethical review. Data collected contained no personal identifiers.

\section{Results}

\section{Samples}

Between 25 October, 2010 and 23 December, 2011, a blood sample was obtained from each of 2,585 nonpregnant individuals attending the Sittwe and Thetkalpyin clinics in Rakhine State and suspected to have malaria infection. Baseline characteristics of the included population are shown in Table 3.

Microscopy and RDT results are shown in Table 1: 531/ 2,585 (20.5\%) patients were infected with P. falciparum, $587 / 2,585(22.7 \%)$ with $P$. vivax or P. malariae. and $1,337 /$ 2,585 (51.7\%) tested negative; 130 (5.0\%) mixed infections (i.e., six from Sittwe, 124 from Thetkalpyin) were found

Table 1 Malaria diagnosis: microscopy compared with rapid diagnostic test SD Bioline Malaria Ag P.f/Pan 05FK60

\begin{tabular}{|c|c|c|c|c|c|}
\hline \multirow[b]{2}{*}{ Microscopy result } & \multirow[b]{2}{*}{$\begin{array}{l}\text { No. of } \\
\text { samples }\end{array}$} & \multicolumn{4}{|c|}{ SD Bioline Malaria Ag P.f/Pan (05FK60) } \\
\hline & & $\begin{array}{l}\text { Negative (\%) } \\
\text { (control band only) }\end{array}$ & $\begin{array}{l}\text { P. falciparum (\%) } \\
\text { (P. falciparum band only) }\end{array}$ & $\begin{array}{l}\text { Pan* (\%) } \\
\text { (Pan band only) }\end{array}$ & $\begin{array}{l}\text { P.f/Pan (\%) } \\
\text { (P.f and pan band) }\end{array}$ \\
\hline No parasites seen & 1,337 & $1,306(97.7)$ & $13(1.0)$ & $11(0.8)$ & $7(0.5)$ \\
\hline P. falciparum & 531 & $39(7.3)$ & $162(30.5)$ & $13(2.5)$ & $317(59.7)$ \\
\hline P. vivax or P. malariae & 587 & $118(20.1)$ & $3(0.5)$ & $454(77.3)$ & $12(2.0)$ \\
\hline Mixed P.f/pan & 130 & $0(0.0)$ & $6(4.6)$ & $2(1.5)$ & $122(93.9)$ \\
\hline
\end{tabular}

A total of 2,585 blood samples was examined at the Sittwe and Thetkalpyin clinics, Rakhine State, Myanmar.

*SD 05FK60 detects the pan-Plasmodium-specific lactate dehydrogenase (pLDH) expressed in P. falciparum, P. vivax, P. ovale and P. malariae). 
Table 3 Baseline characteristics of the study population

\begin{tabular}{ll}
\hline Male & $1,327(51.3)$ \\
Mean age in years (range) & $10.9(0.1-94)$ \\
Mean temperature in ${ }^{\circ} \mathbf{C}$ (range) & $39.2(35.0-42.0)$ \\
Temperature $\geq \mathbf{3 7 . 5 ^ { \circ } \mathbf { C }}$ & $2,162(85.0)$ \\
Parasitaemia level - & $1,337(51.7)$ \\
Parasitaemia level + & $161(6.2)$ \\
Parasitaemia level ++ & $132(5.1)$ \\
Parasitaemia level +++ & $340(13.2)$ \\
Parasitaemia level ++++ & $615(23.8)$
\end{tabular}

The included population for the study was all non-pregnant patients presenting at the Sittwe and Thetkalpyin clinics, Rakhine State, Myanmar with fever or a history of fever prior 24 hours.

Data are presented as numbers (\%) unless otherwise indicated.

and excluded from the main analysis, only misclassification was assessed

\section{Test performance}

Sensitivity, specificity and predictive values of the SD 05FK60 for detection of malaria are shown in Table 2. The overall sensitivity for $P$. falciparum detection by the SD 05FK60 was 90.2\% (95\% CI: 87.4-92.6) and for P. vivax/ P. malariae 79.4\% (95\% CI: 75.9-82.6). The overall specificity for $P$. falciparum detection by the SD 05FK60 at both clinics was 98.5\% (95\% CI: 97.7-99.1) and for P. vivax/ P. malariae $98.7 \%$ (95\% CI: 97.9-99.2).

The positive predictive value (PPV) for P. falciparum detection by the SD 05FK60 in both projects was $96.0 \%$ (95\% CI: 93.9-97.5\%) and for P. vivax 96.3\% (95\% CI: 94.2$97.8 \%)$ at prevalence levels of $28 \%$ and $31 \%$, respectively. The negative predictive value (NPV) for P. falciparum detection by the SD 05FK60 in both projects was $96.3 \%$ (95\% CI: 94.9-97.5\%) and for P. vivax 92.5\% (95\% CI: 90.794.2\%). Test accuracy indices were calculated separately for the two clinics but no significant difference in performance was observed.
$2.5 \%(13 / 531)$ of the P. falciparum infections were wrongly identified by the SD 05FK60 as non-falciparum infections (Table 1). Of those misclassified two samples had $\mathrm{a}+$, one sample a ++ , four samples $\mathrm{a}+++$ and six samples a ++++ parasitaemia level.

Of the non-falciparum infections 2.5\% (15/587) were misclassified as $P$. falciparum or mixed infection (Table 1): $0.5 \%(3 / 587)$ were positive on the $P$. falciparum band of which one samples had a ++ and one sample ++++ parasitaemia level. 2.0\% (12/587) of the non-falciparum samples were positive on the $P$. falciparum and pan-band of which seven had $\mathrm{a}+++$ and five ++++ parasitaemia level. Of the 130 mixed infections 122 were correctly identified by the SD 05FK60 with a positive $P$. falciparum and panband. Two samples only had a positive pan-band and six samples were only positive on the P. falciparum band.

Table 4 shows the malaria detection rate with SD 05FK60 at different levels of parasitaemia. The sensitivity varied between $90.5 \%$ and $95.8 \%$ for $P$. falciparum specimens and between $69.8 \%$ and $97.7 \%$ with a ++ to ++++ parasitaemia level. The sensitivity increased consistently with increasing parasitaemia for $P$. vivax $/ P$. malariae ( $\mathrm{p}<0.005$ ). 25/408 and 13/420 cases with +++ and ++++ parasitaemia levels, respectively, of $P$. falciparum and non-falciparum malaria were missed by the RDT.

\section{Quality control}

The quality control team at the Shoklo Malaria Research Unit (SMRU) in Mae Sot, Thailand, cross-checked 450 slides (225 negative and 225 positive) from MSF's projects in Myanmar from the year 2010, and 250 slides (125 positives and 125 negatives) from MSF's projects in Eastern Rakhine State in Myanmar from the year 2011.

The overall agreement between the positive results had a kappa value of 0.96 (95\% CI: 0.93; 0.99) for 2010 and 0.91 (95\% CI: $0.85,0.98)$ for 2011, indicating very good agreement. The overall sensitivity was $98.3 \%$ (225/ $229)$ in 2010 and $98.4 \%(123 / 125)$ in 2011; overall

Table 4 Sensitivity by parasitaemia level of the SD 05FK60 rapid diagnostic test

\begin{tabular}{|c|c|c|c|c|}
\hline \multirow[t]{2}{*}{ Parasite density } & \multicolumn{2}{|l|}{ P. falciparum } & \multicolumn{2}{|l|}{ P. vivax/P. malariae } \\
\hline & $\begin{array}{l}\text { Number of RDT positives/ } \\
\text { Total positives by micoscopy }\end{array}$ & Sensitivity \% & $\begin{array}{l}\text { Number of RDT positives/ } \\
\text { Total positives by micoscopy }\end{array}$ & Sensitivity \% \\
\hline+ & $34 / 57$ & 59.7 & $14 / 104$ & 13.5 \\
\hline++ & $62 / 66$ & 93.9 & $44 / 63$ & 69.8 \\
\hline+++ & $134 / 148$ & 90.5 & 153/159 & 96.2 \\
\hline++++ & $249 / 260$ & 95.8 & $255 / 261$ & 97.7 \\
\hline Total & $479 / 531$ & 90.2 & $466 / 587$ & 79.4 \\
\hline Chi-square test for trend & $p<0.05$ & & $p<0.05$ & \\
\hline
\end{tabular}

A total of 2,585 blood samples was examined at the Sittwe and Thetkalpyin clinics, Rakhine State, Myanmar.

Estimated parasite densities: $+=10-90 \mathrm{p} / \mu \mathrm{l},++=100-1,000 \mathrm{p} / \mu \mathrm{l},+++=1,000-10,000 \mathrm{p} / \mu \mathrm{l},++++=>10,000 \mathrm{p} / \mu \mathrm{l}$. N: total number of blood samples; $\mathrm{n}$ : total detected by SD Bioline Malaria Ag P.f/Pan (05FK60). 
specificity was $100 \%(211 / 211)$ in 2010 and $99.1 \%$ (110/ 111) in 2011.

\section{Discussion}

Commercially available HRP-2/pLDH tests have been evaluated previously in Myanmar [29,30] but evaluations of the SD 05FK60 are available only from other areas and were conducted before 2007 [17-22]. The study showed overall good performance for the SD 05FK60 RDT in the identification of malaria infections caused by $P$. falciparum and satisfactory results for non-falciparum infections. The poorer detection and identification of $P$. vivax/ $P$. malariae than that of $P$. falciparum is consistent with other evaluations of the SD 05FK60 and pan-pLDH tests in general [17-22].

However, it should be pointed out that in Round 1 of the WHO/TDR/FIND/CDC evaluation, the panel detection score (PDS) of the SD 05FK60 reported at parasitaemia levels of 200 parasites $/ \mu \mathrm{l}$ was $96.2 \%$ for $P$. falciparum and $35.0 \%$ for $P$. vivax [18]. The same product was retested in Round 3 of this evaluation and showed a slightly decreased PDS of $92.9 \%$ for $P$. falciparum along with an improved detection score of $97.1 \%$ for $P$. vivax [19]. It is unclear which version of the product was received in the programme - the catalogue number always remained the same - and this inconsistency is a commonly encountered problem by MSF when an in vitro diagnostic product is changed.

The sensitivity of the SD 05FK60 improves with increasing parasitaemia. This is a well-known limitation of RDTs. Less expected were the findings that 25 P. falciparum infections and $12 P$. vivax/P. malariae infections with +++ or ++++ parasitaemia were undetected by the SD 05FK60. The non-detection of $P$. falciparum infections at high parasitaemia levels could be explained by the prozone effect [31-33] or by deletion of the histidinerich repeat region in the HRP-2 gene [34]. It was not possible to conduct further molecular analysis of the Plasmodium cohort in the area to undermine this argument. However, this does not explain the false negative pan-pLDH band.

Translating these results into the impact on patients, 52 (10.2\%) of a total 531 P. falciparum mono-infections would have been missed by using the RDT without microscopy. Although the specificity has been high for both $P$. falciparum and non-falciparum infections: $98.5 \%$ and 98.7\% respectively, thirteen infections were misidentified as $P$. vivax/P. malariae malaria and as a result would have been treated with chloroquine, a drug to which P. falciparum is unlikely to be susceptible [35]. Considering the P. vivax/P. malariae cases, 121 out of 587 (20.6\%) individuals would have not received any malaria treatment despite being infected. Only 31 individuals out of a total of
1,337 (2.3\%) would have been unnecessarily treated with anti-malarials on the basis of RDT.

Although the results of the performance of the SD 05FK60 are satisfactory, MSF decided to opt for microscopy as the first choice for malaria diagnosis in its programmes in Rakhine State, Myanmar. This was a pragmatic decision based on the clinicians' greater trust in microscopy, particularly in light of the cases with high parasitaemia that would have been missed if microscopy had not been used in addition to the RDT. MSF reserves RDTs for use in decentralized settings and where a high number of patients precludes microscopy. Therefore MSF decided to continue using microscopy in the two clinics in Rakhine State, Myanmar for the routine diagnosis of malaria, although RDTs have been found to be more cost-effective and more feasible in other settings [36-38].

This study has several limitations. The data were collected as part of routine programmatic activities and were unlinked to patient records. Thus, it was impossible to collect clinical information, such as recent intake of antimalarials or the presence of other factors, which may have influenced the results. Another limitations is that the + system has been used to grade parasitaemia instead of a parasite count. This less accurate estimation may have influenced the results on sensitivity especially in the stratified analysis of parasitaemia levels.

It is important to note that the described data were obtained in true field conditions, i e, conditions that were suboptimal in terms of RDT storage and handling and staff expertise, and non-standardized specimens may have been present. It is known that such factors influence RDT performance $[39,40]$, but it is difficult to optimize all these variables under difficult real-life conditions. However, this is also a strength of the study, as it represents test performance under field conditions.

MSF is able to train and maintain good malaria microscopy in its field settings. However, this may not be possible in a wider public health arena. In 2006 the WHO estimated that, if the recommendation to obtain parasitological confirmation of malaria before prescribing treatment were to be followed, screening would need to be scaled up from 122 million to 780 million tests each year in Southeast Asia alone, a 600\% increase. Such scaling up would undoubtedly be difficult to achieve without the use of simple, reliable RDTs [7].

\section{Competing interests}

The authors declare that they have no competing interests.

\section{Authors' contributions}

WTN organized the sample collection and quality control in Rakhine State and performed the data entry. CSK performed the statistical analysis. CSK, WTN, EP and LS analysed and interpreted the results and drafted the manuscript. All authors approved the final manuscript. 


\section{Acknowledgements}

We thank the MSF staff in Myanmar for their contribution to data collection and for technical support, especially Darli Po Po Aung, MSF laboratory coordinator, and Kyaw Soe, MSF laboratory supervisor, in Myanmar.

\section{Author details}

${ }^{1}$ Médecins Sans Frontières International, Plantage Middenlaan 14, 1018 DD Amsterdam, The Netherlands. ${ }^{2}$ Médecins Sans Frontières Holland, Yangon, Myanmar. ${ }^{3}$ Médecins Sans Frontières Holland, Amsterdam, The Netherlands.

Received: 13 March 2013 Accepted: 10 May 2013

Published: 21 May 2013

\section{References}

1. World Health Organization: Guidelines for the treatment of malaria. 2nd edition. Geneva: WHO; 2010 [http://whqlibdoc.who.int/publications/2010/ 9789241547925_eng.pdf]

2. Roll Back Malaria: Global Malaria Action Plan: for a malaria free world. [http:// www.rollbackmalaria.org/gmap/gmap.pdf

3. World Health Organization: The use of malaria rapid diagnostic tests. [http:// whqlibdoc.who.int/publications/2004/9290610883.pdf]

4. Wongsrichanalai C, Barcus MJ, Muth S, Sutamihardja A, Wernsdorfer WH: A review of malaria diagnostic tools: microscopy and rapid diagnostic test (RDT). Am J Trop Med Hyg 2007, 77(Suppl 6):119-127.

5. Moody A: Rapid diagnostic tests for malaria parasites. Clin Microbiol Rev 2002, 15:66-78.

6. Murray CK, Gasser RA Jr, Magill AJ, Miller RS: Update on rapid diagnostic testing for malaria. Clin Microbiol Rev 2008, 21:97-110.

7. Bell D, Peeling R, WHO Regional Office for the Western Pacific/TDR: Evaluation of rapid diagnostic tests: malaria. Nat Rev Microbiol 2006 4(9 Suppl):S34-S38.

8. World Health Organization: Parasitological confirmation of malaria diagnosis, Report of a WHO technical consultation. Geneva; 2009 [http://whqlibdoc. who.int/publications/2010/9789241599412_eng.pdf]

9. Feachem RGA, Phillips AA, Hwang J, Cotter C, Wielgosz B, Greenwood BM, Sabot O, Rodriguez MH, Abeyasinghe RR, Ghebreyesus TA, Snow RW: Shrinking the malaria map: progress and prospects. Lancet 2010, 376:1566-1578.

10. Zarocostas J: Malaria treatment should begin with parasitological diagnosis where possible, says WHO. BMJ 2010, 340:C1402.

11. Reyburn H: New WHO guidelines for the treatment of malaria. BMJ 2010, 340:c2637.

12. Makler MT, Piper RC, Milhous WK: Lactate dehydrogenase and the diagnosis of malaria. Parasitol Today 1998, 14:376-377.

13. Piper R, Lebras J, Wentworth L, Hunt-Cooke A, Houze S, Chiodini P, Makler M: Immunocapture diagnostic assays for malaria using Plasmodium lactate dehydrogenase (pLDH). Am J Trop Med Hyg 1999, 60:109-118.

14. World Health Organization Global Malaria Programme: Good practices for selecting and procuring rapid diagnostic tests for malaria. [http://whqlibdoc. who.int/publications/2011/9789241501125 eng.pdf]

15. World Health Organization: List of known commercially available antigendetecting malaria RDTs information for national public health services and UN Agencies wishing to procure RDTs. [http://www.wpro.who.int/malaria/ internet/resources.ashx/RDT/docs/MD table34\%2B\%281\% 29_totallistofISO131485criteria.pdf]

16. President's Malaria Initiative: Malaria Operational Plan — FY2011. Greater Mekong Sub-Region. [http://www.pmi.gov/countries/mops/fy11/ mekong_mop-fy11.pdf]

17. Van der Palen M, Gillet P, Bottieau E, Cnops L, Van Esbroeck M, Jacobs J: Test characteristics of two rapid antigen detection tests (SD FK50 and SD FK60) for the diagnosis of malaria in returned travelers. Malar $J$ 2009, 8:90

18. WHO/FIND/CDC/TDR: Malaria Rapid Diagnostic Test Performance, Results of WHO product testing of malaria RDTs: Round 1. 2008 [http://www.who.int/ tdr/publications/tdr-research-publications/rdt-performance/en/index.html]

19. WHO/FIND/CDC/TDR: Malaria Rapid Diagnostic Test Performance, Results of WHO product testing of malaria RDTs: Round 3 (2010-2011). [http://www. who.int/tdr/publications/documents/rdt3.pdf]

20. Abba K, Deeks JJ, Olliaro P, Naing CM, Jackson SM, Takwoingi Y, Donegan S, Garner P: Rapid diagnostic tests for diagnosing uncomplicated $P$. falciparum malaria in endemic countries. Cochrane Database Syst Rev 2011, 7:CD008112.

21. Dev $\mathrm{V}$ : Relative utility of dipsticks for diagnosis of malaria in mesoendemic area for Plasmodium falciparum and $P$. vivax in northeastern India. Vector Borne Zoonotic Dis 2004, 4:123-130.

22. Ratsimbasoa A, Randriamanantena A, Raherinjafy R, Rasoarilalao N, Ménard D: Which malaria rapid test for Madagascar? Field and laboratory evaluation of three tests and expert microscopy of samples from suspected malaria patients in Madagascar. Am J Trop Med Hyg 2007, 76:481-485

23. Bell D, Perkins MD: Making malaria testing relevant: beyond test purchase. Trans R Soc Trop Med Hyg 2008, 102:1064-1066.

24. Rennie W, Phetsouvanh R, Lupisan S, Vanisaveth V, Hongvanthong B Phompida S, Alday P, Fulache M, Lumagui R, Jorgensen P, Bell D, Harvey S: Minimising human error in malaria rapid diagnosis: clarity of written instructions and health worker performance. Trans $R$ Soc Trop Med Hyg 2007, 101:9-18.

25. Gillet P, Maltha J, Hermans V, Ravinetto R, Bruggeman C, Jacobs J: Malaria rapid diagnostic kits: quality of packaging, design and labelling of boxes and components and readability and accuracy of information inserts. Malar J 2011, 10:39.

26. Chinkhumba J, Skarbinski J, Chilima B, Campbell C, Ewing V, San Joaquin M, Sande J, Ali D, Mathanga D: Comparative field performance and adherence to test results of four malaria rapid diagnostic tests among febrile patients more than five years of age in Blantyre, Malawi. Malar J 2010, 9:209.

27. World Health Organization/FIND: Methods Manual for Laboratory Control Testing of Malaria Rapid Diagnostic Tests. [http://www.wpro.who.int/malaria/ NR/rdonlyres/461C306D-D720-43CA-A476-1A250EC3C26A/0/ rdt laboratory qc testing_meth_man_v6.pdf]

28. World Health Organization: Malaria Microscopy Quality Assurance Manual. Version 1. Geneva: WHO; 2009 [http://www.who.int/malaria/publications/ malaria_microscopy_QA_manual.pdf]

29. Ashley EA, Touabi M, Ahrer M, Hutagalung R, Htun K, Luchavez J, Dureza C, Proux S, Leimanis M, Lwin M, Koscalova A, Comte E, Hamade P, Page AL, Nosten F, Guerin PJ: Evaluation of three parasite lactate dehydrogenasebased rapid diagnostic tests for the diagnosis of falciparum and vivax malaria. Malar J 2009, 8:241.

30. Xiaodong S, Tambo E, Chun W, Zhibin C, Yan D, Jian W, Jiazhi W, Xiaonong Z: Diagnostic performance of CareStartTM malaria HRP2/pLDH (Pf/pan) combo test versus standard microscopy on falciparum and vivax malaria between China-Myanmar endemic borders. Malar J 2013, 12:6.

31. Gillet P, Mori M, Van Esbroeck M, Van den Ende J, Jacobs J: Assessment of the prozone effect in malaria rapid diagnostic tests. Malar J 2009, 8:271.

32. Gillet $P$, Scheirlinck A, Stokx J, De Weggheleire A, Chaúque HS, Canhang OD, Tadeu BT, Mosse CD, Tiago A, Mabunda S, Bruggeman C, Bottieau E, Jacobs J: Prozone in malaria rapid diagnostics tests: how many cases are missed? Malar J 2011, 10:166

33. Luchavez J, Baker J, Alcantara S, Belizario V Jr, Cheng Q, McCarthy JS, Bell D: Laboratory demonstration of a prozone-like effect in HRP2-detecting malaria rapid diagnostic tests: implications for clinical management. Malar J 2011, 10:286

34. Koita OA, Doumbo OK, Ouattara A, Tall LK, Konaré A, Diakité M, Diallo M, Sagara I, Masinde GL, Doumbo SN, Dolo A, Tounkara A, Traoré I, Krogstad DJ: False-negative rapid diagnostic tests for malaria and deletion of the histidine-rich repeat region of the hrp2 gene. Am J Trop Med Hyg 2012, 86:194-198.

35. Smithuis F, Shahmanesh M, Kyaw MK, Savran O, Lwin S, White NJ: Comparison of chloroquine, sulfadoxine/pyrimethamine, mefloquine and mefloquine-artesunate for the treatment of falciparum malaria in Kachin State. North Myanmar. Trop Med Int Health. 2004, 9:1184-1190.

36. Batwala V, Magnussen P, Hansen KS, Nuwaha F: Cost-effectiveness of malaria microscopy and rapid diagnostic tests versus presumptive diagnosis: implications for malaria control in Uganda. Malar J 2011, 10:372.

37. Batwala V, Magnussen P, Nuwaha F: Comparative feasibility of implementing rapid diagnostic test and microscopy for parasitological diagnosis of malaria in Uganda. Malar J 2011, 10:373.

38. D'Acremont V, Kahama-Maro J, Swai N, Mtasiwa D, Genton B, Lengler C: Reduction of anti-malarial consumption after rapid diagnostic tests implementation in Dar es Salaam: a before-after and cluster randomized controlled study. Malar J 2011, 10:107. 
39. Jorgensen P, Chanthap L, Rebueno A, Tsuyuoka R, Bell D: Malaria rapid diagnostic test in tropical climates: the need for a cool chain. Am J Trop Med Hyg 2006, 74:750-754

40. Chiodini PL, Bowers K, Jorgensen P, Barnwell JW, Grady KK, Luchavez J, Moody AH, Cenizal A, Bell D: The heat stability of Plasmodium lactate dehydrogenase-based and histidine-rich protein 2-based malaria rapid diagnostic tests. Trans R Soc Trop Med Hyg 2007, 101:331-337.

doi:10.1186/1475-2875-12-167

Cite this article as: Kosack et al:: Routine parallel diagnosis of malaria using microscopy and the malaria rapid diagnostic test SD 05FK60: the experience of Médecins Sans Frontières in Myanmar. Malaria Journal 2013 12:167.

\section{Submit your next manuscript to BioMed Central and take full advantage of:}

- Convenient online submission

- Thorough peer review

- No space constraints or color figure charges

- Immediate publication on acceptance

- Inclusion in PubMed, CAS, Scopus and Google Scholar

- Research which is freely available for redistribution 